\title{
Higher Order Singularities in Piecewise Linear Vector Fields
}

\author{
Xavier Tricoche, Gerik Scheuermann, Hans Hagen \\ Computer Science Department, University of Kaiserslautern, Germany
}

Summary. Piecewise linear interpolation of 2D scattered vector data is a classical, simple and fast scheme to process the discrete information provided by experiments or numerical simulations. Nevertheless, its major drawback is its low order that prevents satisfying approximation of non linear behaviors. For topology-based methods in particular, commonly applied in vector field visualization, it often restricts the structural features found to very few possible configurations which may be insufficient for interpretation. In this paper, on the contrary, we consider piecewise linear vector fields from the modeling viewpoint, showing that they can exhibit arbitrary complex topological features.

\section{Introduction}

Piecewise linear vector fields are used in many applications. Their simple mathematical description ensures a very good insight into the topological structure. For scientific visualization in particular, it is the most suited way to handle $2 \mathrm{D}$ scattered vector data for the depiction of the corresponding flow: the given positions are first associated with a triangulation and a linear interpolation is then processed in each triangle resulting in a vector field continuous over the domain. The computation of streamlines (or integral curves) can then be done very accurately in the phase plane by using analytic formulas. Unfortunately, this simplicity usually restricts the encountered features to very few possible behaviors (locally linear) which deprives the extracted structure of most topological configurations existing in analytic vector fields. The qualitative analysis of vector fields on the plane has been a subject of major interest in pure and applied mathematics in this century. In [4], Poincaré laid the foundations of this field. A major contribution was then the work of Andronov ([1]). Topology concepts were next introduced in scientific visualization in [2]: It was shown that focusing on the singularities of a vector field leads to a synthetic depiction of the corresponding flow. The deficiencies of the piecewise linear interpolant for that purpose is a problem that has been considered in Scientific Visualization in the last years. In [6], an approximation method was used to enable the appearance of higher order singular points in piecewise linear vector fields by transforming the field in domains made up of neighboring triangles containing several critical points. The mathematics involved referred to Clifford algebra. An higher order method was also introduced in [5] to automatically detect flow features called vortex core lines. 
Furthermore, the lack of smoothness of piecewise linear interpolation motivated the application of Nielson's $C^{1}$-interpolant for vector field topology depiction (see [7]).

In this paper, we propose an overview of $2 \mathrm{D}$ vector field topology taken from the qualitative theory of second order dynamic systems, then we show that complicated singularities may also occur in piecewise linear vector fields and propose a method to model and properly analyze them. The paper is thus structured as follows. First, we introduce the basic notions required for the qualitative analysis of vector fields. Fundamental theorems are given that enable the definition of possible sector types for a singular point in the general case. Second, we focus on linear vector fields, exposing the few singularity configurations that may appear in this special case. Third, we consider piecewise linear interpolation: we show how to model any type of topological sector and conversely how to depict the structure of complicated topological features.

\section{Topology of 2D Vector Fields}

\subsection{Definitions and Fundamental Theorems}

In the following, we consider a steady vector field defined on the plane.

Definition 1. A steady planar vector field is a map

$$
\begin{aligned}
v: \mathbb{R}^{2} & \longrightarrow T \mathbb{R}^{2} \simeq \mathbb{R}^{2} \\
X & \longmapsto v(X)
\end{aligned}
$$

that is, a map that associates a $2 \mathrm{D}$-vector value with each point on the plane.

Practically, the vector field will be analyzed through its integral curves (also called orbits or paths).

Definition 2. An integral curve through a point $X_{0} \in \mathbb{R}^{2}$ of a vector field $v: \mathbb{R}^{2} \longrightarrow \mathbb{R}^{2}$ is a map

$$
\alpha_{X_{0}}: \mathbb{R} \supset I \longrightarrow \mathbb{R}^{2}
$$

where

$$
\left\{\begin{array}{l}
\dot{\alpha}_{X_{0}}(t)=v\left(\alpha_{X_{0}}(t)\right), \quad \forall t \in I \\
\alpha_{X_{0}}(0)=X_{0}
\end{array}\right.
$$

One supposes that the considered vector field is continuous and satisfies the Lipschitz condition around each point of the plane.

Definition 3. The continuous vector field $v: \mathbb{R}^{2} \longrightarrow \mathbb{R}^{2}$ is said to satisfy the Lipschitz condition around each point of $\mathbb{R}^{2}$ if their exists $K>0$ such that

$$
\forall(X, Y) \in \mathbb{R}^{2} \times \mathbb{R}^{2}, \quad\|v(X)-v(Y)\|<K\|X-Y\|
$$

If this condition is true, then $K$ is called the Lipschitz constant of v. 
Remark 1. In the case of a piecewise linear vector field defined on a bounded triangulation, the Lipschitz condition is satisfied. As a matter of fact, the field is $C^{\infty}$ (being affin linear) over each triangle and thus satisfies the Lipschitz condition over each triangle. Let $K_{i}$ be a Lipschitz constant corresponding to the $i$ th triangle and consider the following configuration: One has:

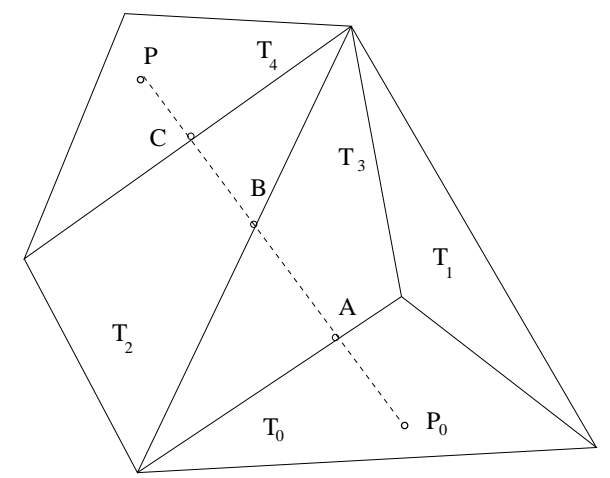

Fig. 1. Lipschitz constant in the piecewise linear case

$$
\begin{aligned}
\left\|v(P)-v\left(P_{0}\right)\right\| \leq & \|v(P)-v(C)\|+\|v(C)-v(B)\|+\|v(B)-v(A)\| \\
& +\left\|v(A)-v\left(P_{0}\right)\right\| \\
\leq & K_{4} \times\|P-C\|+K_{2} \times\|C-B\|+K_{3} \times\|B-A\| \\
& +K_{0} \times\left\|A-P_{0}\right\| \\
\leq & \left(K_{0}+K_{2}+K_{3}+K_{4}\right)\left\|P-P_{0}\right\| \\
\leq & \left(\sum_{i=0}^{N} K_{i}\right)\left\|P-P_{0}\right\|
\end{aligned}
$$

where $N$ is the total number of triangles. So if one sets $K=\sum_{i=0}^{N} K_{i}, K$ is a Lipschitz constant of the vector field for the whole triangulated domain and one verifies the hypotheses in the following theorems.

Theorem 1. Let $v: \mathbb{R}^{2} \longrightarrow \mathbb{R}^{2}$ be a continuous vector field satisfying the Lipschitz condition around each point of $\mathbb{R}^{2}$. Then there exists a unique integral curve through any $X_{0} \in \mathbb{R}^{2}$. Furthermore, every integral curve is defined over $\mathbb{R}$.

Actually, in the theorem above, two different curves through a position $X_{0} \in$ $\mathbb{R}^{2}$ may only differ by the choice of their parameterization. One can then define the phase portrait of the vector field as the family of all its paths over the plane. A fundamental property for the study of the structure of a vector field is given in the following theorem. 
Theorem 2. Let $v$ be as before and let $\alpha$ and $\beta$ be two integral curves of $v$ on the closed interval $\left[t_{0}, t_{1}\right]$. Then, for all $t \in\left[t_{0}, t_{1}\right]$ one has

$$
\|\alpha(t)-\beta(t)\| \leq\left\|\alpha\left(t_{0}\right)-\beta\left(t_{0}\right)\right\| \exp \left(K\left(t-t_{0}\right)\right)
$$

That is, one has continuity of integral curves with respect to initial conditions. In the domain of study, one distinguishes two types of point:

Definition 4. A singular point (also called equilibrium state) of a vector field $v$ is a point at which the field is zero.

It results from the uniqueness of the integral curve of $v$ through a point that singular points are the only locations on the plane where two different integral curves can meet. A point which is not singular is said to be regular.

Now, one focuses on the topology of the vector field, that is, geometrically, the structure of its integral curves. As shown in [1], the knowledge of the singular points of the field provides a very good characterization of the phase portrait topology.

\subsection{Singular Points Analysis}

All the results in this section are taken from [1]. One should consult this reference for a more rigorous understanding of these topics.

Center Type First consider the case of singular points that are approached by no integral curve. Such singularities are said to be of center type. In this case, on can find a neighborhood of the singular point where all paths are closed, inside one another, and contain the singular point in their interior.

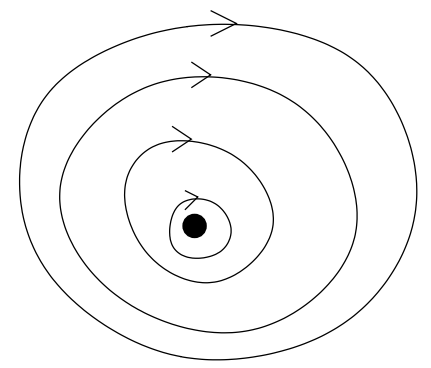

Fig. 2. center

Non Center Type In this case, one has not only a single path converging to the singular point but actually at least two. To analyze the local structure of such a point, consider the following configuration: where $L_{M}^{*}$ and $L_{M^{\prime}}^{*}$ are 


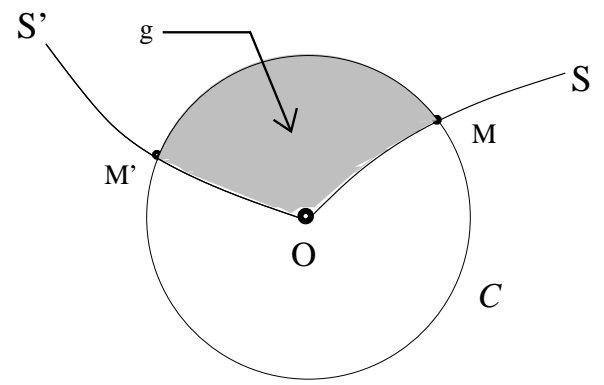

Fig. 3. curvilinear sector

(positive or negative) semi-paths tending to $O . C$ is a circle with arbitrary small fixed radius and $g$ is the (open) region bounded by the integral curves $O M$ and $O M^{\prime}$ and the arc of $M M^{\prime}$ on $C$, called curvilinear sector.

The structure of a singular point of non center type is characterized by the behavior of the integral curves passing through points of one of its sectors. In fact, there exist three different types of curvilinear sectors.

- Case 1. If $L_{M}^{*}$ tends to 0 for $t \longrightarrow \infty$ and if $L_{M^{\prime}}^{*}$ tends to 0 for $t \longrightarrow$ $-\infty$ and if every integral curve passing through the open $g$ leaves $g$ for both $t \longrightarrow \infty$ and $t \longrightarrow-\infty$, the sector will be called a hyperbolic or saddle sector. In this case, both $L_{M}^{*}$ and $L_{M^{\prime}}^{*}$ are called separatrices of

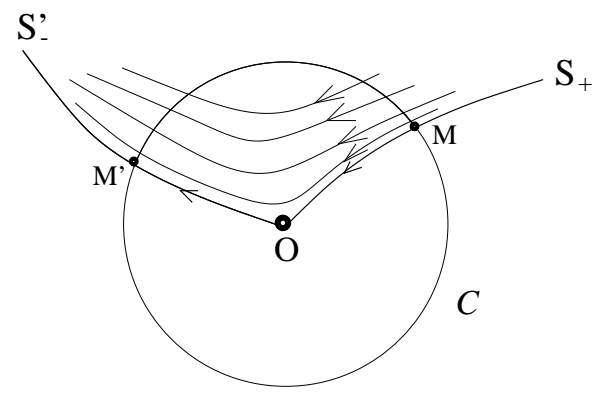

Fig. 4. hyperbolic sector

the singular point $O$ (more precisely, $L_{M}^{*}$ is an $\omega$-separatrix and $L_{M^{\prime}}^{*}$ is an $\alpha$-separatrix).

- Case 2. If $L_{M}^{*}$ and $L_{M^{\prime}}^{*}$ both tend to $O$ for $t \longrightarrow \infty$ (resp. $t \longrightarrow-\infty$ ) and if every integral curve through the open $g$ tends to 0 for $t \longrightarrow \infty$ (resp. $t \longrightarrow-\infty)$ without leaving $g$ and leaves $g$ for $t \longrightarrow-\infty$ (resp. $t \longrightarrow \infty)$, the sector is known as an $\omega$ - (resp. $\alpha$-) parabolic sector. 


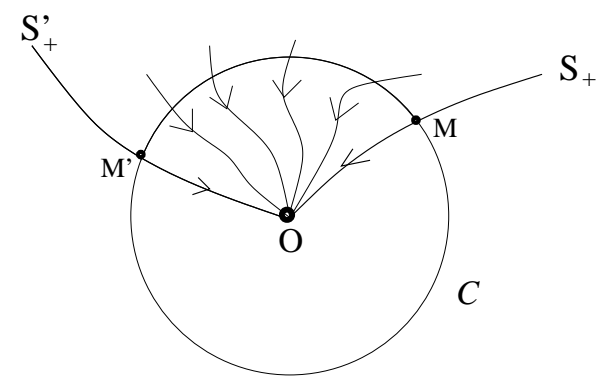

Fig. 5. parabolic sector

- Case 3. If $L_{M}^{*}$ and $L_{M^{\prime}}^{*}$ are two semi-paths on the same path and if all the paths through a point inside this loop form nested loops tending to $O$ for both $t \longrightarrow \infty$ and $t \longrightarrow-\infty$, the sector is called an elliptic sector.

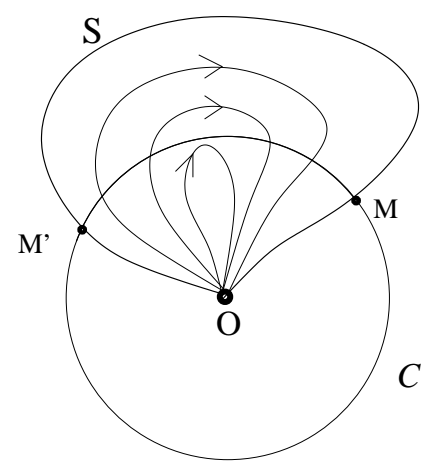

Fig. 6. elliptic sector

Consequently, any singular point may be characterized by the type, angular location, and number of its curvilinear sectors. The precise meaning of this characterization is described in the following.

Theorem 3. If the structures of two singular points are related through a one-to-one correspondence between their respective $\omega$-separatrices, $\alpha$-separatrices and elliptic regions then there exists a path-preserving topological mapping of a neighborhood of the first onto a neighborhood of the second preserving orientation and direction of $t$.

\section{Piecewise Linear Interpolation}

After having considered singular points from a general viewpoint, we now focus on their possible structure in the special case of a piecewise linear vector 
field. We start with a review of the few basic topological features of a linear vector field before presenting a method that makes use of the "flexibility" introduced by the piecewise linearity to model any topological structure for a singular point.

\subsection{Linear Interpolation}

An affin linear vector field $v$ is described in the following way

$$
v(X)=A X+b
$$

where

$$
\begin{aligned}
& X=\left(\begin{array}{l}
x \\
y
\end{array}\right) \\
& A=\left(\begin{array}{ll}
\alpha_{X} & \beta_{X} \\
\alpha_{Y} & \beta_{Y}
\end{array}\right) \text { and } b=\left(\begin{array}{l}
\gamma_{X} \\
\gamma_{Y}
\end{array}\right)
\end{aligned}
$$

(If $v$ has a zero, then one takes its location as new coordinates origin and thus considers the linear field $\left.v^{\prime}(X)=A X\right)$.

An affin linear vector field is uniquely determined by its Jacobian (or gradient matrix) at the location of its possible zero. That is, depending on the eigenvalues of the matrix $A$, integral curves of $v$ may have different aspects over the plane. The following classification is taken from [3].

- Case 1. A has real eigenvalues of opposite signs. The zero is called a saddle point.

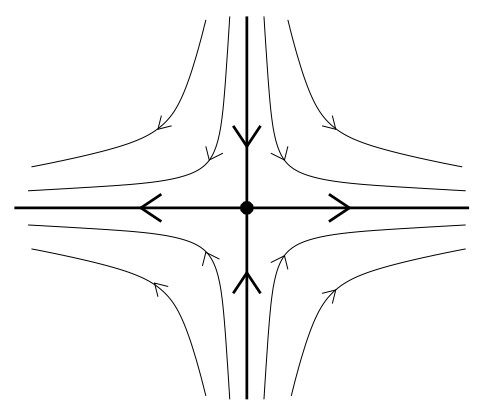

Fig. 7. saddle point

- Case 2. All eigenvalues have negative real parts. The zero is called a sink, because any integral curve tends to $O$ for $t \longrightarrow \infty$.

- Case 2a. $A$ is diagonalizable and its eigenvalues are different. The zero is called a node sink. The special case where the eigenvalues are equal is called a focus sink. 


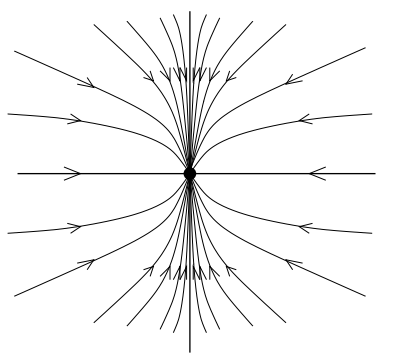

Fig. 8. node sink

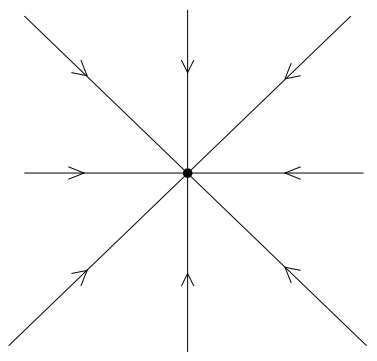

Fig. 9. focus sink

- Case 2b. $A$ is not diagonalizable but has one real negative eigenvalue. The zero is called an improper node sink.

- Case 2c. $A$ has two complex conjugate eigenvalues with negative real parts. The zero is called a spiral sink.

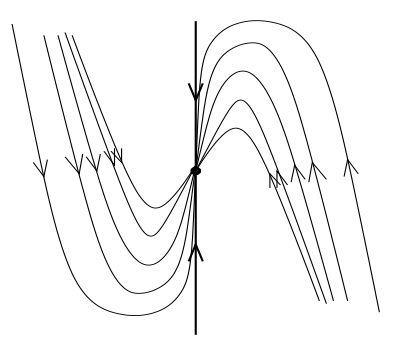

Fig. 10. improper node sink

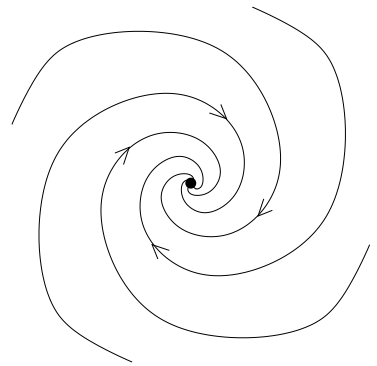

Fig. 11. spiral sink

- Case 3. All eigenvalues have positive real parts. The zero is called a source, because any integral curve tends to it for $t \longrightarrow-\infty$.

- Case 3a. $A$ is diagonalizable and its eigenvalues are different. The zero is a node source. If both eigenvalues are equal, the zero is a focus source.

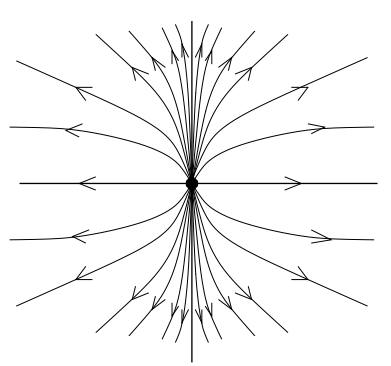

Fig. 12. node source

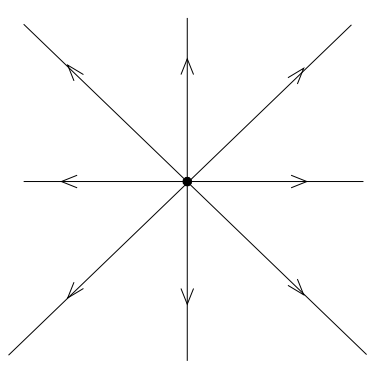

Fig. 13. focus source 
- Case 3b. A is not diagonalizable but has one real positive eigenvalue. The zero is called an improper node source.

- Case 3c. $A$ has two complex conjugate eigenvalues with positive real parts. The zero is called a spiral source.

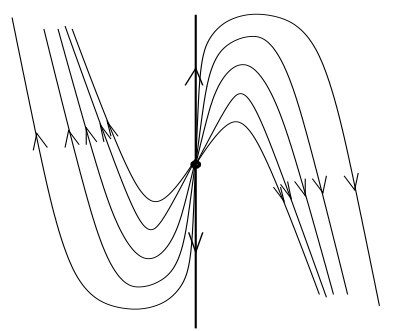

Fig. 14. improper node source

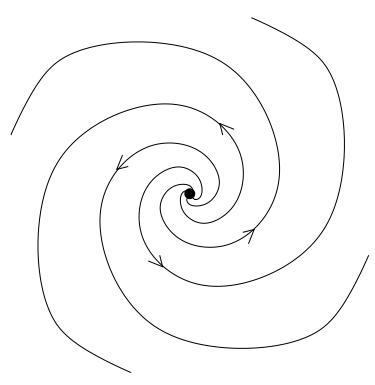

Fig. 15. spiral source

- Case 4. A has pure imaginary eigenvalues. The zero is then called a center (see Fig. 2).

Thus, in a linear vector field, the only singularities that can be found are (possibly after a coordinate change) of the one of the eight configurations above. Such singular points are said to be simple or of first order.

\subsection{Higher Order Singular Points}

So what is new, from the topological viewpoint, with piecewise linear interpolation?

One is given a triangulation of planar points associated with vector values and inside each triangle cell, one builds a linear combination of the three vectors at the vertices. So inside each triangle, the vector field is linear and if a singularity exists in its interior, it has the topological structure of one of the cases above. But the situation becomes fundamentally different when the singularity is to be found on a triangle vertex. Indeed, in this case, there is no neighborhood of the singular point lying in the definition domain of a linear field (i.e. one single triangle). Consequently, the analysis of the Jacobian matrix is of no help in finding out the topological structure of such a singular point. Fundamentally, one has to face an arbitrary singular point (i.e. a singular point in the neighborhood of which a linear approximation may no longer be possible). In analytic vector fields, such singular points correspond to points on the plane at which the Jacobian matrix has not full rank. Simple examples are given by the so-called monkey-saddle $\left(v(x, y)=\left(x^{2}-y^{2},-2 x y\right)\right)$ and dipole $\left(v(x, y)=\left(x^{2}-y^{2}, 2 x y\right)\right)$. In the following, we first show how to model any type of singularity (that is the number, positions and natures of 


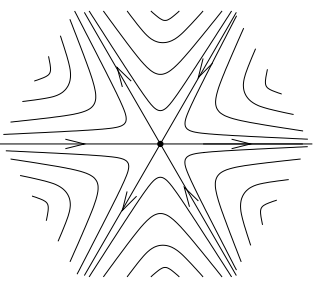

Fig. 16. monkey saddle

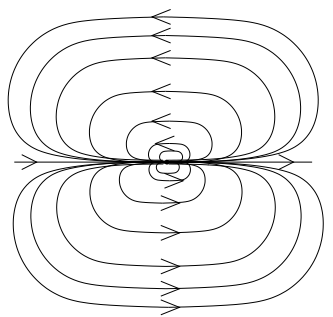

Fig. 17. dipole

the curvilinear sectors are arbitrary defined) and then we focus on the converse problem, detecting the local topological structure of a given singularity lying at a triangulation vertex.

Modeling of Singular Points In this section, one is dealing with the following problem (see theorem 3): Given a list of angles $\left(\omega_{i}\right)_{i=1, \ldots, n 1}$ of $\omega$ separatrices, a list of angles $\left(\alpha_{i}\right)_{i=1, \ldots, n 2}$ of $\alpha$-separatrices, and a list $\left(g_{i}\right)_{i=1, \ldots, n 3}$ of elliptic sectors, build a piecewise linear vector field that presents an equivalent singular point. Here, the problem should be subdivided into modeling a single curvilinear sector starting and stopping at prespecified angular positions, given by the sorted values of the angles introduced above.

Consequently, one considers the three possible sector type cases.

Remark: In the following, the neighborhood of the singular point is actually the set of triangles that are incident to the considered "singular" vertex.

Hyperbolic Sector A hyperbolic sector is bounded by two separatrices of opposite kind (an $\omega$ - and an $\alpha$-separatrix). Consider an $\omega$-separatrix located at $\theta=\omega$ and an $\alpha$-separatrix located at $\theta=\alpha$. Building a triangle as in Fig. 18, and setting $\boldsymbol{v}_{1}=-\boldsymbol{u}_{\omega}$ and $\boldsymbol{v}_{2}=+\boldsymbol{u}_{\alpha}$, one gets the expected hyperbolic behavior for all integral curves starting inside the triangle $A B C$.

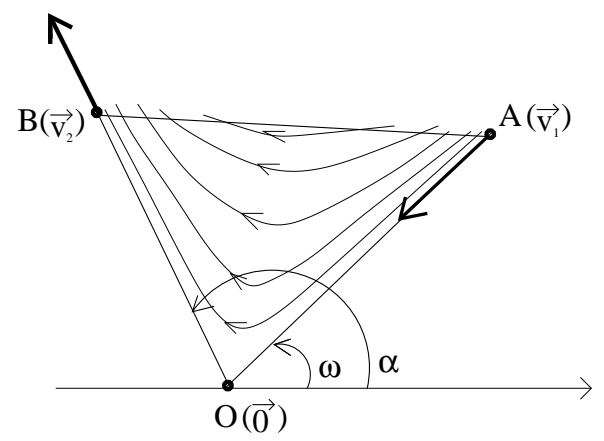

Fig. 18. piecewise linear hyperbolic sector 
Parabolic Sector According to the definition above, a parabolic sector is bounded by two separatrices of the same kind (both $\omega$ - or both $\alpha$-separatrices). Consider two $\omega$-separatrices located at $\theta=\omega_{1}$ and $\theta=\omega_{2}$ respectively. Building a triangle as in Fig. 19, and setting $\boldsymbol{v}_{1}=-\boldsymbol{u}_{\omega_{1}}$ and $\boldsymbol{v}_{2}=-\boldsymbol{u}_{\omega_{2}}$, one gets the expected parabolic behavior for all integral curves starting inside the triangle $A B C$.

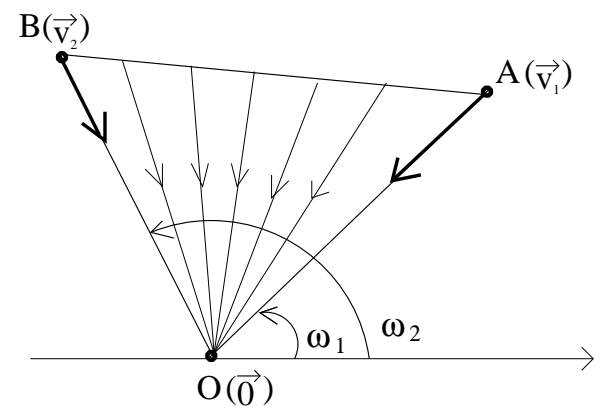

Fig. 19. piecewise linear parabolic sector

Elliptic Sector In this case, one is not given two angle coordinates of two bounding separatrices but a curvilinear sector bounded by a loop integral curve that tends to $O$ for both $t \longrightarrow \infty$ and $t \longrightarrow-\infty$. The modeling of such a sector by a piecewise linear vector field requires the curve itself to be described in terms of its tangential directions for $t \longrightarrow \infty$ and $t \longrightarrow-\infty$. Consider Fig. 20.

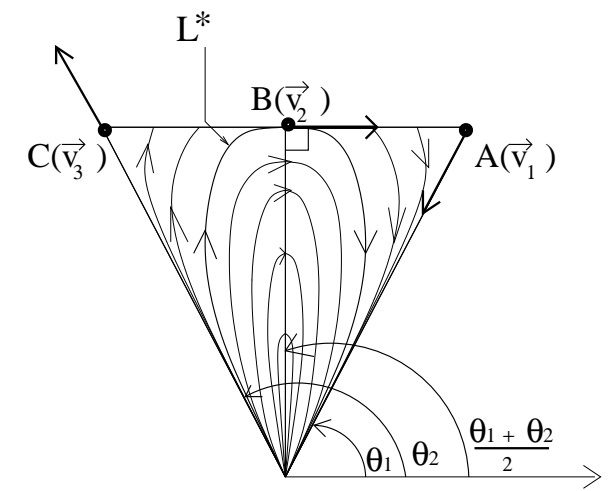

Fig. 20. piecewise linear elliptic sector

The angle coordinates $\theta_{1}$ and $\theta_{2}$ are the tangential direction of the loop curve $L^{*}$ that bounds the modeled elliptic sector. The vector values at $A$ and $C$ are set as in the hyperbolic case. From the former case, one knows that the linear sector defined by $A, B$ and $O$ is hyperbolic. This means that an elliptic sector cannot be met in a linear field. To build such a sector, one has 
thus to split the triangle into two sub-triangles. By setting the vector value at the corresponding additional point $B$ as shown above, one gets the expected elliptic behavior for all integral curves through points located inside the loop $L^{*}$.

Fig. 21 illustrates the possible cases.

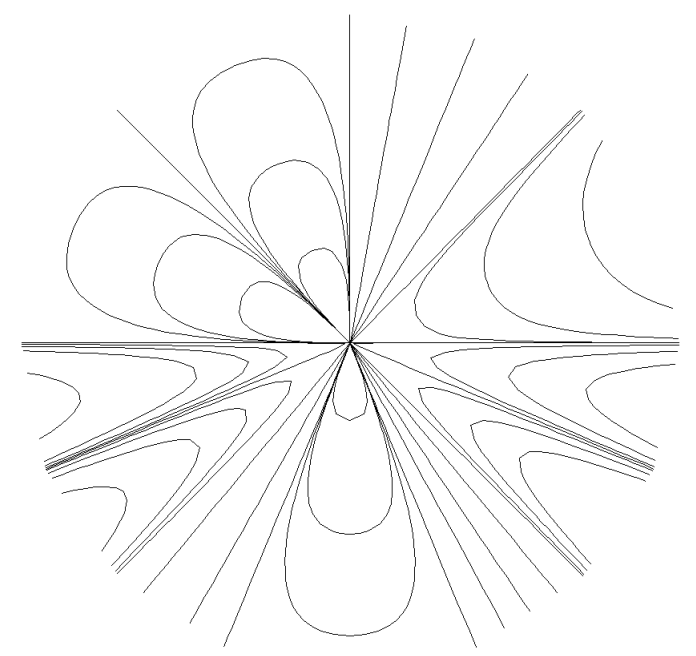

Fig. 21. modeled singular point

Local Topology Detection Conversely, suppose that a "piecewise linear" singular point is given, that is a zero vector located at a vertex of a piecewise linear interpolated triangulation. One wants to locate and analyze the different topological sectors of such a singular point. According to what precedes, it consists in seeking the boundary curves of hyperbolic sectors (called separatrices) and the different sets of nested loop curves tending to the singular point for both increasing and decreasing time.

The following lemma will be very useful for the processing.

Lemma 1. In the neighborhood of a singular point lying on a vertex of a piecewise linear interpolated triangulation, the angle coordinate of the vector field does not depend on the distance to the singular point.

Proof: Consider the situation in Fig. 22. In the triangle $A B C$, the vector value at $P$ is linear interpolated between $O$ and $Q$. One thus gets

$$
\begin{aligned}
\boldsymbol{v}(P) & =x \boldsymbol{v}(Q)+(1-x) \boldsymbol{v}(O) \\
& =x * \boldsymbol{Q}
\end{aligned}
$$




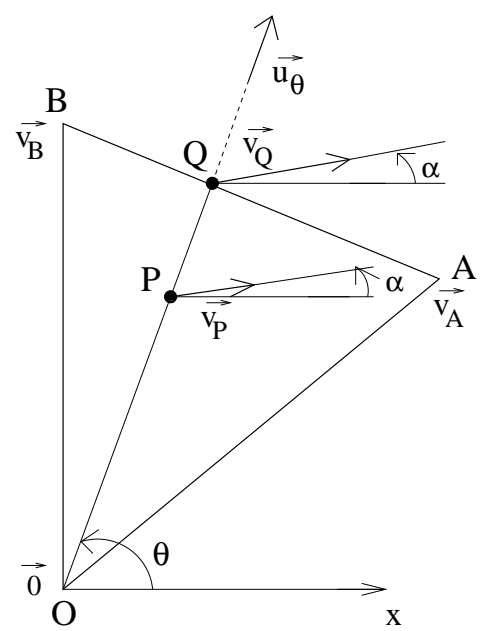

Fig. 22. Angular coordinate

That is, $\boldsymbol{v}(P)$ is collinear to $\boldsymbol{v}(Q)$ and thus, taking $O$ as coordinate origin, both vectors have the same angle coordinate. Q.E.D.

In the remaining, one calls their common angular coordinate $\boldsymbol{v}(\theta)$.

Using this property, one can locate the angular positions of all separatrices by looking for angles where the vector field is collinear to the coordinate vector and checking the type of the sectors on both sides to find out if one has actually found a separatrix. Seeking the angular coordinates where the vector field is orthogonal to the coordinate vector enables next the distinction between a hyperbolic and an elliptic sector (see section 20). To simplify the results, one adopts the following notations: at the angular coordinates where the vector field is orthogonal to the coordinate vector, one distinguishes angles where the cross-product $\boldsymbol{u}_{\theta} \wedge \boldsymbol{v}(\theta)$ is positive (called orthogonal + ) from those where it is negative (called orthogonal-). At the angular coordinates where the vector field is parallel to the coordinate vector, one distinguishes angles where the scalar product $\boldsymbol{u}_{\theta} \cdot \boldsymbol{v}(\theta)$ is positive (called parallel+) from those where it is negative (called parallel-). (see Fig. 23).

One gets then the graph proposed in Fig. 24 for the determination of a sector type.

\section{Conclusion}

A piecewise linear interpolation of $2 \mathrm{D}$ vector data is a simple and fast way to reconstruct the information provided by simulations or experiments into a continuous vector field. Nevertheless, this scheme is often regarded as insufficient for the detection of complex topological features because of its low 


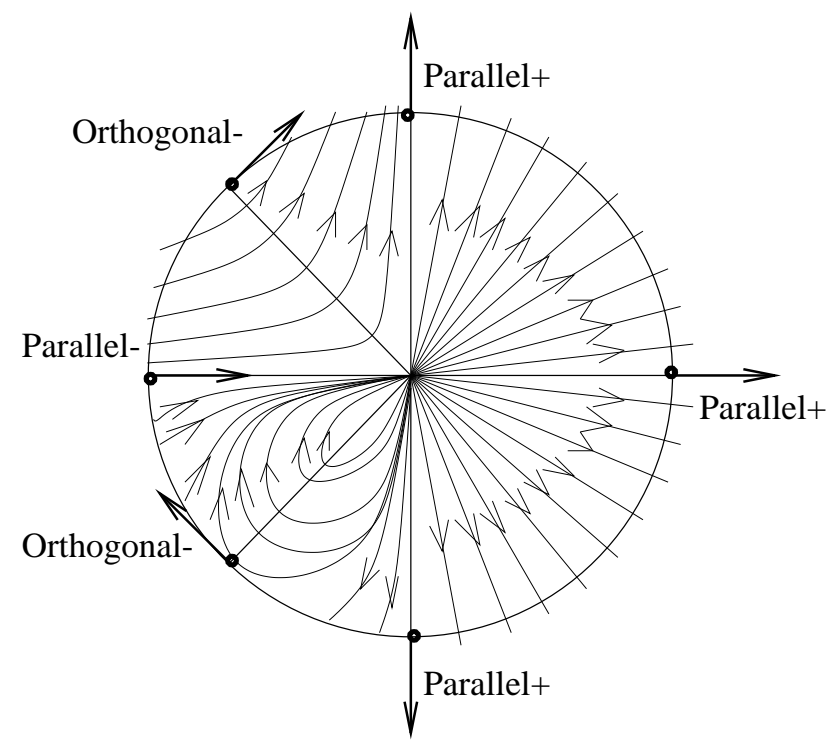

Fig. 23. remarkable positions
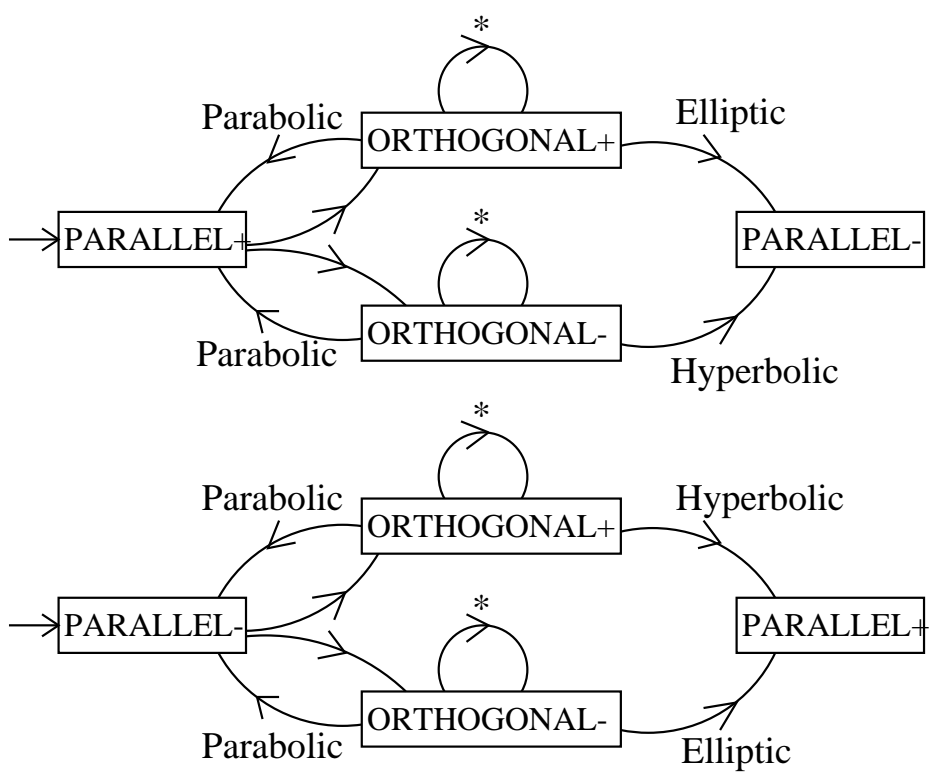

Fig. 24. sector type determination graph 
degree. Yet, we have shown in this paper that a zero vector value located at a vertex position results in a complex topological structure in its neighborhood. Actually, one has shown that any possible topological behavior of a vector field may be thus encountered and not only the few basic features existing in linear fields. An interesting application of this result has been considered which is the modeling of singularities of any type and complexity in piecewise linear vector fields.

\section{References}

[1] Andronov A.A., Leontovich E.A., Gordon I.I., Maier A.G., Qualitative Theory of Second-Order Dynamic Systems Israel Program For Scientific Translation, Halsted Press, 1973.

[2] Helman J.L., Hesselink L., Representation and Display of Vector Field Topology in Fluid Flow Data Sets. IEEE Computer, 1989.

[3] Hirsch M.W., Smale S., Differential Equations, Dynamical Systems and Linear Algebra. Academic Press, 1974.

[4] Poincaré H., Sur les courbes définies par une équation différentielle Oeuvres, Vol. I., Paris, Gauthier-Villars, 1928.

[5] Roth M., Peikert R., A Higher-Order Method For Finding Vortex Core Lines. IEEE Visualization, 1998, Research Triangle Park, NC.

[6] Scheuermann G., Krüger H., Menzel M., Rockwood A.P., Visualizing NonLinear Vector Field Topology. IEEE Transactions On Visualization \& Computer Graphics, Vol. 4, No. 3, July 1998.

[7] Scheuermann G., Tricoche X., Hagen H., C1-Interpolation for Vector Field Topology Visualization. IEEE Visualization, 1999, San Francisco, CA. 